\title{
Editorial
}

\section{Symmetry and Group Theory and Its Application to Few-Body Physics}

\author{
Xiao-Yan Gu, ${ }^{1}$ Fabien Gatti, ${ }^{2}$ Shi-Hai Dong, ${ }^{3}$ and Jian-Qiang Sun ${ }^{4}$ \\ ${ }^{1}$ Department of Physics, East China University of Science and Technology, Shanghai 200237, China \\ ${ }^{2}$ CTMM, Institut Charles Gerhardt, UMR 5253, Universite Montpellier II, Place Eugene Bataillon, 34095 Montpellier, France \\ ${ }^{3}$ Departamento de Física, Escuela Superior de Física y Matemáticas, Instituto Politécnico Nacional, UPALM, Edificio 9, \\ 07738 México, DF, Mexico \\ ${ }^{4}$ College of Information Science and Technology, Hainan University, Haikou 570228, China
}

Correspondence should be addressed to Jian-Qiang Sun; sunjq123@qq.com

Received 31 August 2014; Accepted 31 August 2014; Published 18 December 2014

Copyright (C) 2014 Xiao-Yan Gu et al. This is an open access article distributed under the Creative Commons Attribution License, which permits unrestricted use, distribution, and reproduction in any medium, provided the original work is properly cited.

Symmetry and group theory are important tools in analyzing physics and mechanics problems, which possess abstract theory and wide application. This issue complies 10 exciting papers, most of which investigate character solutions of differential system and oscillator.

Symmetries, conservation laws, and solutions of differential systems are analyzed. G. G. Polat et al. study invariant solutions for classical mechanics problems of Lienard-type by partial Noether and $\lambda$-symmetry approaches and give Lie point symmetry group analysis and $\lambda$-symmetry classification of the fin equation. Conservation law and exact solution of the modified Hunter-Saxton equation by the nonlocal conservation method and multiplier approach are analyzed by S. San and E. Yaşar. Symmetry and traveling wave solution of Boussinesq equation are obtained by L. D. Moleleki and C. M. Khalique based on Lie symmetry method.

The oscillator is a class of important model of few-body physics. J. Sadeghi et al. obtain the energy spectrum and general wave function of the oscillator with Aharonov-Casher system by the factorization method. The modified raising and lowering operators of quantum harmonic oscillators with twisted algebra are obtained by J. Naji et al.

Lie group method, which is proposed based on Lie group and Lie algebra theory, is an important method in solving the differential equation on manifold. J.-Q. Sun et al. solve the diffusion equation by Lie group method. M. Ávila et al. obtain scales of time where the quantum discord allows an efficient execution of the DQC1 algorithm.
Constructing the shift operators for infinite circular and spherical wells using the potential group approach is analyzed by G.-H. Sun et al. These shift operators depend on all spatial variables of quantum systems and connect some eigenstates of confined systems of different radii $R$ sharing energy levels with a common eigenvalue. B. Lasorne presents how a formulation based on Lie group homomorphisms can simplify the treatment of unitary similarity transformations of Hamiltonian matrices in nonadiabatic photochemistry.

By compiling these papers, we hope to enrich our readers and researchers knowledge with respect to symmetry and group theory and its application to few-body physics.

Xiao-Yan Gu Fabien Gatti Shi-Hai Dong Jian-Qiang Sun 


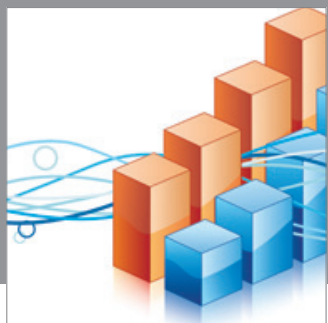

Advances in

Operations Research

mansans

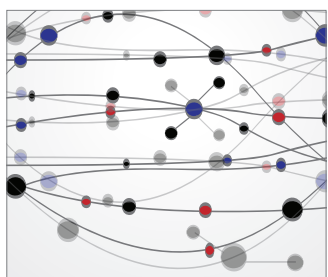

The Scientific World Journal
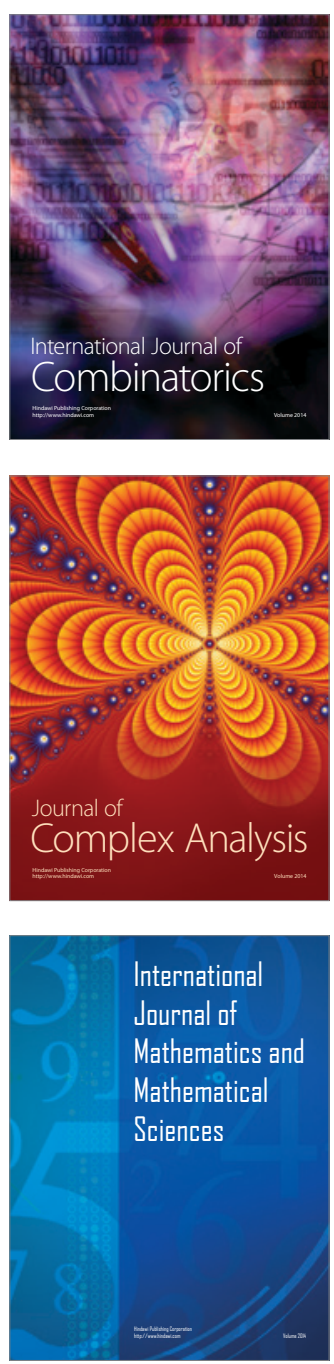
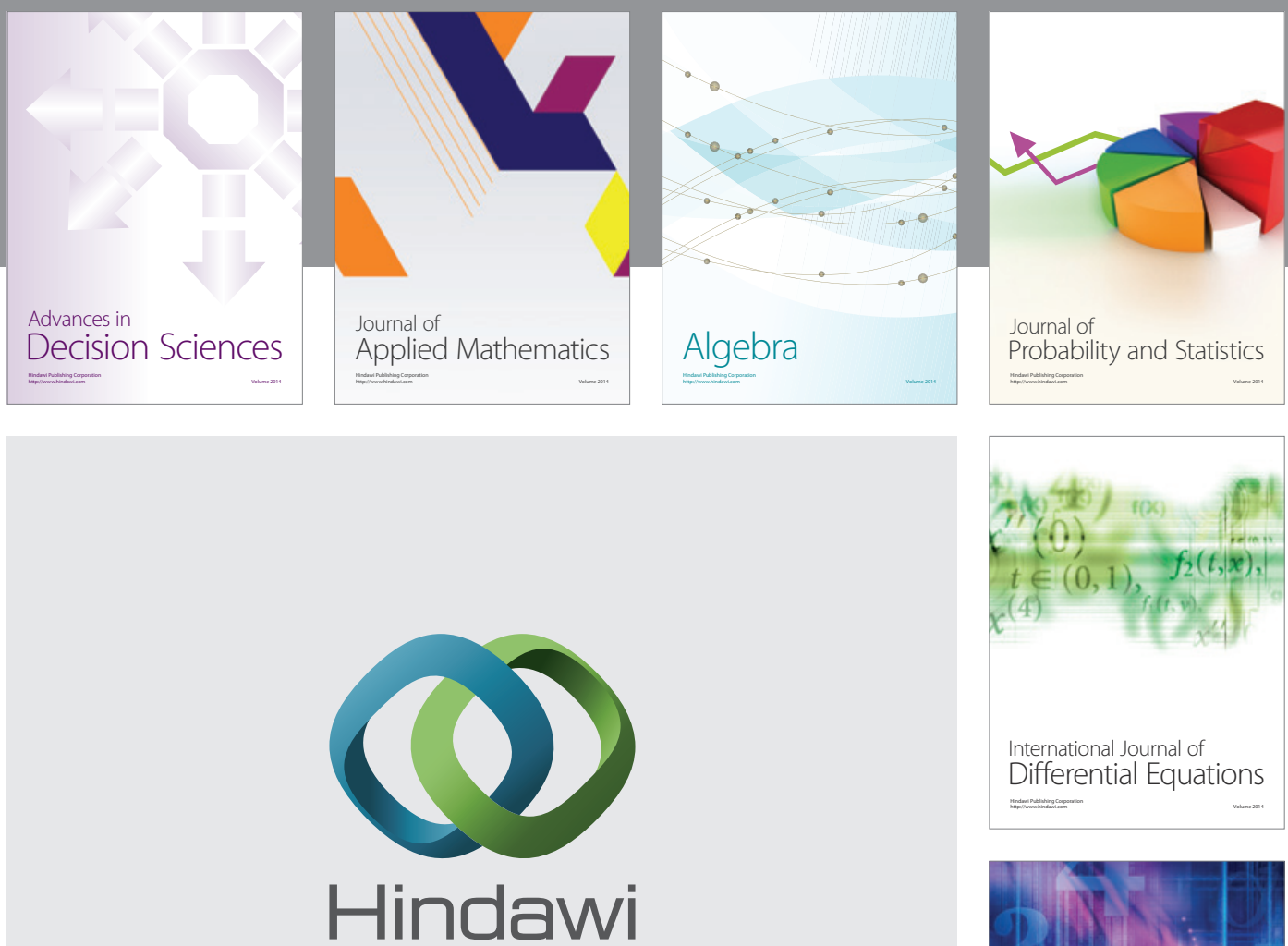

Submit your manuscripts at http://www.hindawi.com
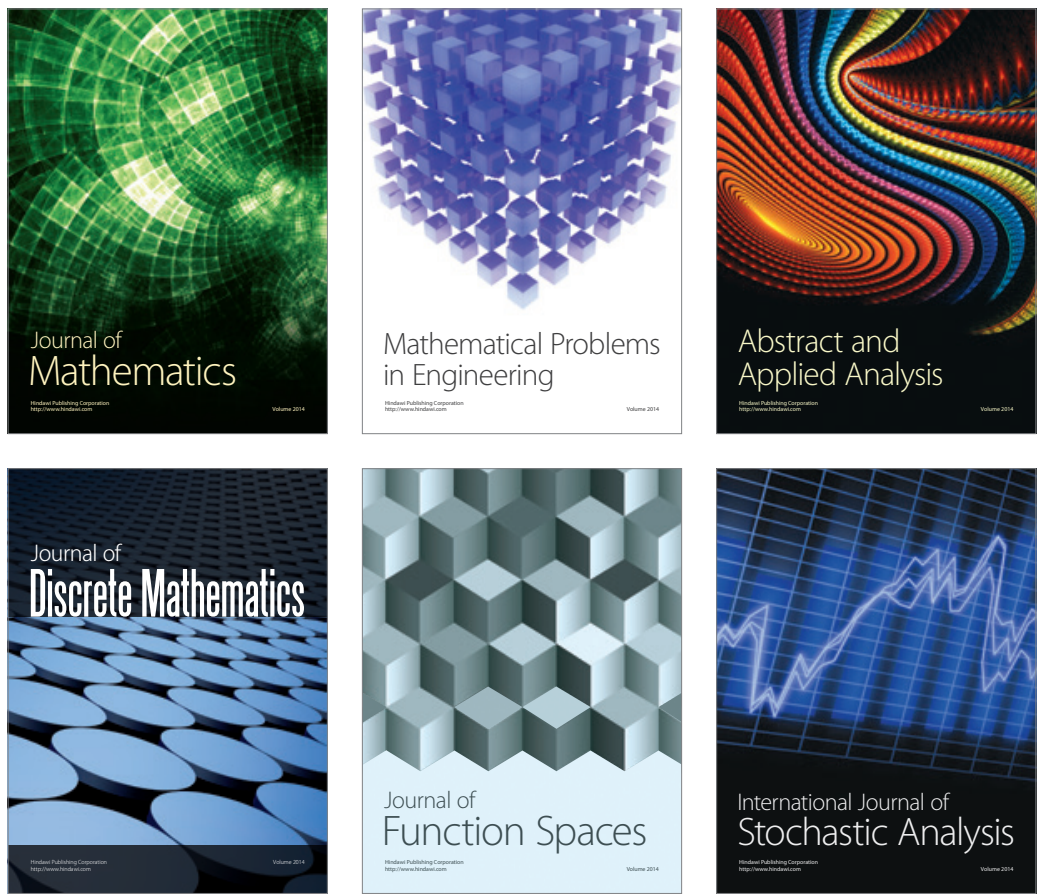

Journal of

Function Spaces

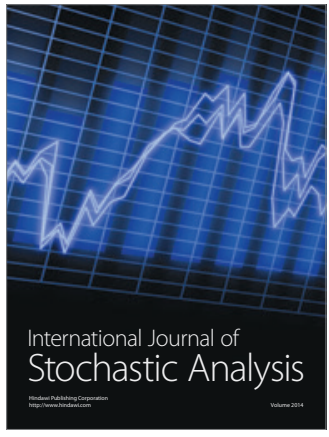

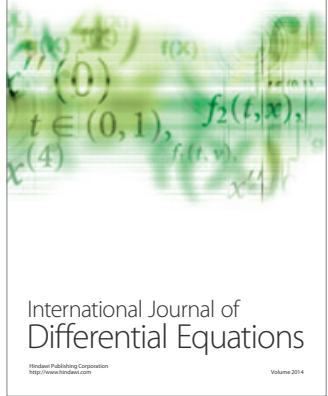
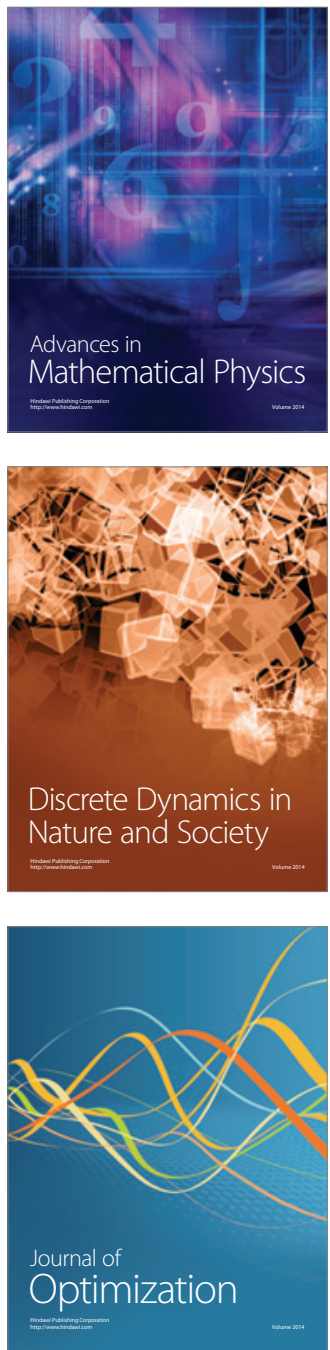